\title{
ISOFLAVONAS DE SOJA E PROPRIEDADES BIOLÓGICAS
}

\author{
CLAUDIO LIMA AGUIAR *
}

\begin{abstract}
A presente revisão teve como objetivo discutir algumas das propriedades biológicas relacionadas às isoflavonas encontradas em soja. Esses compostos são conhecidos por suas atividades biológicas tais como, estrogênica, antifúngica, antioxidante e antitumoral (mama e próstata). Além disso, inibem a ação de enzimas ligadas à divisão celular, sendo suas atividades biológicas mais acentuadas nas formas agliconas que glicosiladas. Tem sido demonstrado que as isoflavonas podem agir contra muitas doenças, devendo ser realizados outros estudos para avaliar a aplicação desses compostos na alimentação humana.
\end{abstract}

PALAVRAS-CHAVE: ISOFLAVONAS; SOJA.

\section{ISOFLAVONAS}

Sabe-se que flavonóides de frutas, vegetais e soja (Glycine max Merrill), como as isoflavonas, em suas diferentes formas, desempenham importante papel no corpo humano. Podem agir como antioxidantes, anti-inflamatórios, antimicrobianos entre outras atividades biológicas, tornando os produtos que os contém em alimento funcional ou nutracêutico.

A soja está entre os alimentos funcionais e embora tenha sido mencionada somente em 1941 nas estatísticas oficiais de produção de grãos do Rio Grande do Sul, a primeira referência sobre seu cultivo no Brasil data de 1882 (VERNETTI, 1977). Sua composição química, com base em $100 \mathrm{~g}$ de amostra seca, constitui-se de $40 \mathrm{~g}$ de proteínas, $30 \mathrm{~g}$ de glicídios, $20 \mathrm{~g}$ de lipídios, $226 \mathrm{mg}$ de cálcio, $546 \mathrm{mg}$ de fósforo e 8,8 $\mathrm{mg}$ de ferro (WOLF e COWAN, 1971; SGARBIERI et al., 1981). Além da proteína, a soja fornece os ácidos graxos linoléico e linolênico (NAWAR, 1985) e algumas vitaminas (FRANCO, 1986). O grão intacto de soja pode conter além de carboidratos, ácidos graxos (83\% de ácidos graxos insaturados), proteínas e minerais, $4 \%$ de saponinas, $5 \%$ de lecitinas, $\beta$-sitosteróis, $\alpha$-tocoferóis e inibidores de protease, embora as isoflavonas despertem maior interesse devido às suas propriedades biológicas.

* $\quad$ MSc., Pesquisador Colaborador, Laboratório de Bioquímica, Faculdade de Engenharia de Alimentos, Universidade Estadual de Campinas, Campinas, SP.

(e-mail: claguiar@yahoo.com.br). 
As isoflavonas são compostos pertencentes ao grupo dos flavonóides, que se caracterizam por apresentar estrutura polifenólica, com dois anéis de benzeno $(A)$ ligados a um terceiro anel $(B)$ na posição do carbono 3 (Figura 1).

\section{FIGURA1- ESTRUTURA QUÍMICA DE UMA ISOFLAVONA ENCONTRADA EM SOJA}<smiles>O=c1c(-c2ccc(O)cc2)coc2cc(O)cc(O)c12</smiles>

O total de isoflavonas encontrado na soja distribui-se, basicamente, em isoflavonas glicosiladas e isoflavonas agliconas (AHLUWALIA et al., 1953). Por outro lado, ELDRIDGE (1982) e FUKUTAKE et al. (1996) afirmaram que genistina e daidzina são os principais isoflavonóides e constituem de 50 a $90 \%$ dos flavonóides da farinha de soja. Outros derivados desses compostos glicosilados foram identificados e compreendem as formas acetil e malonil. PARK et al. (2001b), analisando diferentes cultivares de soja da mesma região brasileira, observaram grande variação na concentração dessas isoflavonas (considerando o teor de isoflavonas totais). Segundo CARRÃO-PANIZZI et al. (1998) a concentração de isoflavonas em soja é geneticamente determinada e afetada por fatores ambientais e pela temperatura local. Segundo GENOVESE e LAJOLO (2001) os fatores que afetam a composição de isoflavonas em soja estão também relacionados à (1) variedade e condições de cultivo, (2) condições de processamento da soja, ou ainda (3) metodologias de análise. Esse último fator constitui etapa importante na avaliação do teor de isoflavonas em soja, tanto pela eficiência do método de extração quanto da própria análise (atualmente baseados principalmente na cromatografia a líquido de alta eficiência). As estruturas químicas de algumas dessas isoflavonas, glicosiladas ou agliconas são exemplificadas na Figura 2.

Já existem muitos produtos como bolos, chocolates e biscoitos elaborados com farinha de soja, que apresentam isoflavonas glicosiladas na sua composição (BARNES et al., 1994; NAWAR, 1985), ou ainda, na forma de acetil ou malonil isoflavonas. A forma malonil isoflavona é transformada a glicosil isoflavona durante o processamento da soja para extração do óleo em temperaturas acima de $100^{\circ} \mathrm{C}$. KUDOU et al. (1991) relataram que a extração alcoólica à quente promove a transformação de malonil 
isoflavona mediante desesterificação às formas b-glicosiladas (Figura 3). PARK et al. (2001a) observaram que a $120^{\circ} \mathrm{C}$ parte das malonil isoflavonas foram convertidas a seus conjugados glicosilados.

\section{FIGURA 2 - ESTRUTURA QUÍMICA DE ISOFLAVONAS GLICOSILADAS E AGLICONAS ENCONTRADAS EM SOJA}

\begin{tabular}{|c|c|c|c|c|c|}
\hline Estrutura molecular plana & Isoflavona & $R 1$ & $R 2$ & $R 3$ & $R 4$ \\
\hline & Daidzina & O-glicosil & $\mathrm{H}$ & $\mathrm{H}$ & $\mathrm{OH}$ \\
\hline & Daidze na & $\mathrm{OH}$ & $\mathrm{H}$ & $\mathrm{H}$ & $\mathrm{OH}$ \\
\hline & Glicitina & O-glicosil & $\mathrm{OCH}_{2}$ & $\mathrm{H}$ & $\mathrm{OH}$ \\
\hline & Glicite na & $\mathrm{OH}$ & $\mathrm{OCH}_{2}$ & $\mathrm{H}$ & $\mathrm{OH}$ \\
\hline & Genistina & O-glicosil & $\mathrm{H}$ & $\mathrm{OH}$ & $\mathrm{OH}$ \\
\hline & Geniste na & $\mathrm{OH}$ & $\mathrm{H}$ & $\mathrm{OH}$ & $\mathrm{OH}$ \\
\hline
\end{tabular}

\section{FIGURA 3 - DESESTERIFICAÇÃO DE MALONIL ISOFLAVONA À $\beta$ - GLICOSIL ISOFLAVONA}

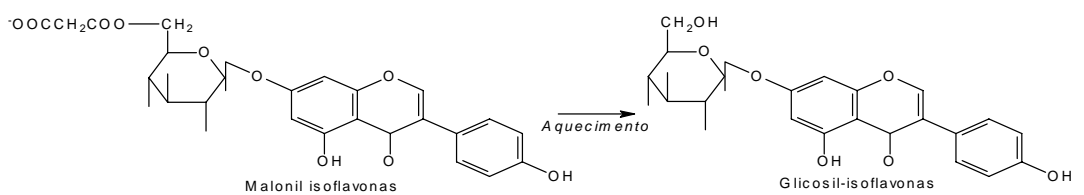

As transformações entre os isômeros de isoflavonas podem ser observadas pela aplicação de enzimas específicas, como a $\beta$-glicosidase ou a lactase phorizin hidrolase, encontradas no intestino delgado de mamíferos. IKEDA et al. (1995), PARK et al. (2001a) e PARK et al. (2001c) reportaram que concentrações de isoflavonas agliconas em soja fermentada foram maiores quando comparadas com soja não-fermentada. Provavelmente, devido ao fato das glicosil isoflavonas terem sido hidrolisadas à agliconas durante fermentação pela transformação enzimática com $\beta$-glicosidase.

\section{ATIVIDADES BIOLÓGICAS}

Isoflavonas são conhecidas por suas propriedades biológicas, incluindo atividade estrogênica (especialmente sobre os sintomas da menopausa e da osteoporose) (MURPHY, 1982), antifúngica (NAIM et al., 1974), 
bactericida, antimutagênica (MIYAZAWA et al., 1999) e antitumoral, especialmente cânceres de mama e próstata (COWARD et al., 1993; PETERSON e BARNES, 1993; DENIS et al., 1999; HIROTA et al., 2000). São essenciais para a sobrevivência de plantas leguminosas, protegendoas contra fitopatógenos pela ação antifúngica. Conforme outros estudos as isoflavonas apresentariam atividades anti-hemolítica e antioxidante, as quais foram observadas em diversos trabalhos encontrados na literatura (ESAKI et al., 1999a; ESAKI et al., 1998; SHAHIDI et al., 1992).

\subsection{ATIVIDADE ANTIOXIDANTE}

Os produtos de soja, tradicionalmente usados no Japão (tais como miso, natto e tempeh), são ativos contra a peroxidação lipídica devido à presença de isoflavonas que estariam ligadas à atividade antioxidante. Além disso, foi reportado que a atividade antioxidante de genisteína ou de outras isoflavonas agliconas foram superiores às de glicosil isoflavonas (ONOZAWA et al., 1998). Os isoflavonóides glicosilados podem ser transformados por processos enzimáticos em agliconas como daidzeína e genisteína, cujas atividades antioxidantes têm sido superiores às das formas glicosiladas (daidzina e genistina), conforme demonstrado por PARK et al. (2001a).

ESAKI et al. (1999b) afirmaram que complexo antioxidante foi isolado de soja fermentada com Aspergillus saitoi e Aspergillus oryzae, ou seja, 6-hidroxidaidzeína (6-OHD), 8-hidroxidaidzeína (8-OHD) e 8hidroxigenisteína (8-OHG). Essas isoflavonas, as quais apresentam estrutura O-dihidroxi entre as posições 7 e 8 ou posições 7 e 6 , foram obtidas de daidzeína e genisteína, respectivamente, por reação de hidroxilação durante o processo fermentativo. Foi também observado que essas formas mostraram atividade antioxidante superior à apresentada por daidzeína e genisteína.

\subsection{ATIVIDADE ANTITUMORAL}

Isoflavonas são conhecidas também por sua atividade antitumoral (mama e próstata), a qual é mais acentuada nas formas agliconas que glicosiladas assim como ocorre com a atividade antioxidante. Muito embora os mecanismos pelos quais as isoflavonas inibem a carcinogênese não sejam conhecidos (SHERTZER et al., 1999), seus efeitos citotóxicos podem estar relacionados à inibição de enzimas como a topoisomerase II (SKIBOLA e SMITH, 2000). As isoflavonas ainda interferem na ação da DNA-topoisomerase II, S6-quinase ribossomal, fosfoinositidío 3-quinase (PI 3-quinase) e proteína quinase C (PKC), enzimas ligadas ao ciclo, diferenciação e proliferação celulares (GAMET-PAYRASTRE et al., 1999). 
Genisteína, em particular, é potente inibidor da tirosina quinase. A inibição dessas enzimas resultaria na proteção contra diversos cânceres do intestino, da próstata e da mama. Genisteína apresenta grande poder no controle de células cancerosas, inibindo mais o crescimento de células tumorais da próstata humana que sua forma glicosilada (MATSUDA et al., 1994).

O câncer de mama é o mais comum entre as mulheres americanas. Sua incidência em mulheres asiáticas é relativamente menor, devido ao consumo de alimentos à base de soja, principalmente, pela ingestão de genisteína. Segundo LI et al. (1999) genisteína inibiu o crescimento de células cancerosas MDA-MB-231 de mama, regulou a expressão de genes relacionados à apoptose e induziu apoptose. Sendo assim, sugerem que genisteína pode ser eficiente quimioterápico contra o câncer de mama. POOL-ZOBEL et al. (2000) mencionaram que genisteína também reprimiu a proliferação de células cancerosas humanas HT29.

BARNES (1995) observou redução significativa do risco de cânceres de pele e cólon e que o efeito da genisteína no controle da proliferação celular depende do tipo de célula cancerosa.

\subsection{INIBIÇÃO ENZIMÁTICA}

Algumas enzimas, como colagenase e elastase, estão associadas à formação de aneurisma e outras doenças vasculares. Nesses casos percebeu-se, em testes in vitro, que flavonóides podem diminuir a ação de proteases sobre proteínas fibrosas e o acúmulo de proteoglucanas e hialuronanas. Extratos vegetais contendo flavonóides inibiram a atividade de elastase, tripsinase e a-quimotripsinase (SCHRAMM e GERMAN, 1998).

WONG e KEUNG (1997) observaram efeito inibitório de um derivado de daidzeína sobre esterol sulfatase, enzima ligada à evolução do câncer de mama. Foi constatado que daidzeína não afetou a esterol sulfatase, no entanto, seus sulfo-conjugados, daidzeína-4'-O-sulfato e daidzeína-7,4'di-O-sulfato, foram potentes inibidores dessa enzima.

Segundo NAGAO et al. (2000) flavonóides como luteolina, quercetina, ramnetina e floretina inibiram profundamente a atividade de $\beta$-caroteno$15,15^{\prime}$-dioxigenase. Entretanto, isoflavonas mostraram pouco efeito inibitório sobre essa enzima, responsável pelo fornecimento de vitamina A mediante catálise oxidativa do $\beta$-caroteno em duas moléculas de retinal. Outra enzima de grande importância, a F0F1-ATPase/ATP sintase que sintetiza ATP durante a fosforilação oxidativa mitocondrial, pode ser inibida por diferentes isoflavonas como genisteína, biocanina A, daidzeína e 
genistina. Genisteína também foi capaz de inibir a $\mathrm{Na}^{+} / \mathrm{K}^{+}-\mathrm{ATP} a s e$. Esse efeito inibitório para ZHENG e RAMIREZ (2000) poderia estar associado à propriedades citotóxicas. Segundo WONG e KEUNG (1999) daidzeína, genisteína, biocanina $A$ e formononetina inibiram potencialmente a atividade de álcool desidrogenase, que é responsável pela oxidação de 3- $\beta$-hidroxiesteróides e a conversão de 5-pregnen-3,20-diona em progesterona.

Estudos cinéticos mostraram que $\mathrm{H}^{+} / \mathrm{K}^{+}$-ATPase gástrica, enzima ligada à secreção gástrica, foi inibida por diferentes flavonóides, incluindo algumas isoflavonas e que o potencial inibitório depende do número de hidroxilas da molécula (MURAKAMI et al., 1999).

\subsection{EFEITO SOBRE O COLESTEROL E ATIVIDADE ESTROGÊNICA}

Testes clínicos realizados por GARDNER et al. (2001) sugerem o efeito hipocolesterolêmico da proteína de soja. Entretanto, esse pode ser parcialmente atribuído às isoflavonas da soja, já que poucos estudos têm verificado seus efeitos isoladamente. Dietas à base de soja são particularmente atrativas para VIGNA et al. (2000), dado ao seu potencial resultado na redução do colesterol e seus efeitos anti-cancerígenos, além da possível modificação dos sintomas da menopausa. Como exemplo tem-se o estudo realizado por LUCAS et al. (2001), envolvendo cinqüenta e quatro mulheres com elevado teor de colesterol. Tal estudo sugere que a redução do teor de colesterol das mulheres submetidas à dietas a base de proteína de soja, suplementadas ou não com isoflavonas, não depende da suplementação com isoflavona.

LUCAS et al. (2001) sugerem que o consumo de proteína de soja está ligado à redução da hipercolesterolemia. Entretanto, outros componentes como isoflavonas e saponinas também podem estar ligados à redução do colesterol. Segundo MESSINA (2000) a proteína de soja, rica em isoflavonas, supostamente inibe a oxidação de lipoproteína de baixa densidade e teria maior efeito na redução de colesterol que as proteínas de soja com baixo teor de isoflavonas. WANGEN et al. (2001) afirmaram que o consumo de isoflavonas, como constituinte da proteína isolada de soja, resulta em pequeno, mas significativo aumento da atividade contra a hipercolesterolemia pós-menopausa em mulheres. Embora com pequenos resultados é possível que as isoflavonas possam contribuir na diminuição de doenças coronárias, se o consumo for contínuo por alguns anos em conjunção com outras estratégias para a redução do colesterol. Para LOVATI et al. (2000) é comum responsabilizar as isoflavonas pelo maior efeito bioquímico em relação à redução do colesterol.

VAN Der SCHOUW et al. (2000) avaliaram a evidência dos benefícios e 
riscos cardiovasculares associados aos fitoestrógenos (compostos químicos divididos em três principais classes: isoflavonas, coumestanas e lignanas), que desempenham atividade similar ao estrogênio. Para MAKELA et al. (1998) alguns desses compostos (isoflavonas) são agentes estrogênicos por si, podendo atuar como estrógeno endógeno.

MESSINA (2000) sugeriu que a incidência das ondas de calor em mulheres asiáticas é menor que em mulheres ocidentais. Entretanto GREENWOOD et al. (2000) demonstraram a inadequação dos dados para avaliação dos efeitos das isoflavonas sobre o câncer de mama, a perda de cálcio e a secura vaginal. Mesmo que os efeitos na saúde humana não possam ser claramente atribuídos às isoflavonas é nítido que alimentos ou suplementos contendo isoflavonas são capazes de exercer algum efeito fisiológico. Desta forma, as isoflavonas tem sido associadas com a redução de lipoproteínas de baixa densidade e de triglicerídios bem como ao aumento de lipoproteínas de alta densidade.

\title{
3 CONCLUSÃO
}

Certamente a soja constitui importante fonte de isoflavonas (compostos fenólicos heterocíclicos com estrutura similar ao $\beta$-estradiol) e que a ação destes compostos na célula depende do tecido alvo e da forma isomérica em que se encontra. Assim, diferentes estudos mostram que dependendo da forma, aglicona ou conjugada, a atividade biológica será maior ou menor.

\begin{abstract}

\section{SOYBEAN ISOFLAVONES AND BIOLOGICAL PROPERTIES}

The present review had as objective to discuss the biological properties related to the isoflavones found in soybean. These compounds are known by their biological activities as, estrogenic, antifungal, antioxidant and antitumor (breasts and prostate). Besides, inhibit the activity of enzymes linked to cell division, being their biological activities more accentuated in aglycones than glycosides forms. It has been demonstrated that the isoflavones may act against some diseases, but more studies are necessary to evaluate the application of these compounds in human feeding.

KEY-WORDS: ISOFLAVONES; SOYBEAN.
\end{abstract}

\section{REFERÊNCIAS}

1 AHLUWALIA, V.K.; BHASIN, M.M.; SESHADRI, T.R. Isoflavones of soybeans. Current Science, v. 22, p. 263-265, 1953.

2 BARNES, S. Effect of genistein on in vitro and in vivo models of 
cancer. Journal of Nutrition, v. 125, Supl.w, p. 777-783, 1995.

3 BARNES, S.; KIRK, M.; COWARD, L. Isoflavones and their conjugates in soy foods: extraction conditions and analysis by HPLCMass Spectrometry. Journal of Agricultural and Food Chemistry, v. 42, n. 11, p. 2466-2474, 1994.

4 CARRAO-PANIZZI, M.C.; KITAMURA, K.; BELEIA, A.D.; OLIVEIRA, M.C.N. Influence of growth locations on isoflavone contents in Brazilian soybeans cultivars. Breeding Science, v. 48, p. 409-413, 1998.

5 COWARD, L.; BARNES, N.C.; SETCHELL, K.D.R.; BARNES, S. Genistein, daidzein, and their beta-glycoside conjungates: antitumor isoflavones in soybean foods from American and Asian diets. Journal of Agricultural and Food Chemistry, v. 41, p. 1961-1967, 1993.

6 DENIS, L.; MORTON, M. S.; GRIFFITHS, K. Diet and its preventive role in prostatic disease. European Urology, v. 35, n.5-6, p. 377387, 1999.

7 ELDRIDGE, A.C. Determination of isoflavones in soybean flours, protein-concentrates, and isolates. Journal of the American Chemical Society, v. 183, p. 90, 1982.

8 ESAKI, H.; KAWAKISHI, S.; MORIMITSU, Y.; OSAWA, T. New potent antioxidative O-dihydroxyisoflavones in fermented Japanese soybean products. Bioscience, Biotechnology and Biochemistry, v. 63, n. 9, p. 1637-1639, 1999a.

9 ESAKI, H.; WATANABE, R.; ONOZAKI, H.; KAWAKISHI, S.; OSAWA, T. Formation mechanism for potent antioxidative Odihydroxyisoflavones in soybeans fermented with Aspergillus saitoi. Bioscience, Biotechnology and Biochemistry, v. 63, n. 9, p. 851858, $1999 b$.

10 ESAKI, H.; ONOZAKI, H.; MORIMITSU, Y.; KAWAKISHI, S.; OSAWA, T. Potent antioxidative isoflavones from soybeans fermented with Aspergillus saitoi. Bioscience, Biotechnology and Biochemistry, v. 64, n. 2, p. 740-746, 1998.

11 FRANCO, G. Tabela de composição química de alimentos. Rio de Janeiro: Atheneu, 1986. p. 145. 
12 FUKUTAKE, M.; TAKAHASHI, M.; ISHIDA, K.; KAWAMURA, H.; SUGIMURA, T.; WAKABAYASHI, K. Quantification of genistein and genistin in soybeans and soybean products. Food and Chemical Toxicology, v. 34, n. 5, p. 457-461, 1996.

13 GAMET-PAYRASTRE, L.; MANENTI, S.; GRATACAP, M.P.; TULLIEZ, J.; CHAP, H.; PAYRASTRE, B. Flavonoids and the inhibition of PKC and PI 3-kinase. General Pharmacology, v. 32, p. 279-286, 1999.

14 GARDNER, C.D.; NEWELL, K.A.; CHERIN, R.; HASKELL, W.L. The effect of soy protein with or without isoflavones relative to milk protein on plasma lipids in hypercholesterolemic postmenopausal women. American Journal of Clinical Nutrition, v. 73, n. 4, p. 728-735, 2001.

15 GENOVESE, M.I.; LAJOLO, F.M. Isoflavonas da soja: fatores que influem nos tipos e teores em alimentos. Food Ingredients, v. 11, p. 62-64, 2001.

15 GREENWOOD, S.; BARNES, S.; CLARKSON, T.B.; EDEN, J.; HELFERICH, W.G.; HUGHES, C.; MESSINA, M.; SETCHELL, K.D.R. The role of isoflavones in menopausal health: consensus opinion of the North American Menopause Society. The Journal of the North American Menopause Society, v. 7, n. 4, p. 215-229, 2000.

16 HIROTA, A.; TAKI, S.; KAWAII, S.; YANO, M.; ABE, N. 1,1-Diphenyl2-picrylhydrazyl radical-scavenging compounds from soybean miso and antiproliferative activity of isoflavones from soybean miso toward the cancer cell lines. Bioscience, Biotechnology and Biochemistry, v. 64, n. 5, p. 1038-1040, 2000.

17 IKEDA, R.; NAOKAZU, O.; WATANABE, T. Changes of isoflavones at various stages of fermentation in defatted soybeans. Nippon Shokuhin Kagaku Kogaku Kaishi, v. 42, n. 5, p. 322-327, 1995.

18 KUDOU, S.; SHIMOYAMADA, M.; IMURA, T.; UCHIDA, T.; OKUBO, $\mathrm{K}$. A new isoflavone glycoside in soybean seeds (Glycine max Merrill), glycitein 7-O-beta-D-(6"-O-acetyl)-glucopyranoside. Agricultural and Biological Chemistry, v. 55, n. 3, p. 859-860, 1991.

19 LI,Y.; UPADHYAY, S.; BHUIYAN, M.; SARKAR, F.H. Induction of apoptosis in breast cancer cells MDA-MB-231 by genistein. 
Oncogene, v. 18, n. 20, p. 3166-3172, 1999.

20 LOVATI, M.R. et al. Soy protein peptides regulate cholesterol homeostasis in Hep G2 cells. Journal of Nutrition, v. 130, n. 10, p. 2543-2549, 2000.

21 LUCAS, E.A. et al. Ethanol-extracted soy protein isolate does not modulate serum cholesterol in Golden Syrian hamsters: a model of postmenopausal hypercholesterolemia. Journal of Nutrition, v. 131, n. 2, p. 211-214, 2001.

22 MAKELA, S. et al. Inhibition of 17 beta-hydroxysteroid oxidoreductase by flavonoids in breast and prostate cancer cells. Proceedings of the Society for Experimental Biology and Medicine, v. 217, n. 3, p. 310-316, 1998.

23 MATSUDA, S. et al. Hydrolysis of isoflavones in soybean cooked syrup by Lactobacillus casei subsp. rhamnosus IFO 3425. J. Ferment. Bioeng., v. 74, n. 5, p. 301-304, 1994.

24 MESSINA, M. Soyfoods and soybean phyto-oestrogens (isoflavones) as possible alternatives to hormone replacement therapy (HRT). European Journal of Cancer, v. 36, p. 71-77, 2000.

25 MIYAZAWA, M. et al. Antimutagenic activity of isoflavones from soybean seeds (Glycine max Merrill). J. Agric. Food Chem., v. 47, n. 4, p. 1346-1349, 1999.

26 MURAKAMI, S.; MURAMATSU, M.; TOMISAWA, K. Inhibition of gastric $\mathrm{H}^{+}, \mathrm{K}^{+}-$ATPase by flavonoids: a structure-activity study. Journal of Enzyme Inhibition, v. 14, n. 2, p. 151-166, 1999.

27 MURPHY, P.A. Phytoestrogen content of processed soybean products. Food Technology, v. 36, p. 60-64, 1982.

28 NAGAO, A. et al. Inhibition of $\beta$-carotene-15,15'-dioxygenase activity by dietary flavonoids. J. Nut. Biochem., v.11, n.6, p. 348-355, 2000.

29 NAIM, M. et al. Soybean isoflavones. Characterization, determination, and antifungal activity. J. Agric. Food Chem., v. 22, n. 5, p. 806810, 1974.

30 NAWAR, W. W. Lipids. In: FENNEMA, C.R. (Ed.). Food chemistry. 
New York: Marcel Dekker, 1985. p. 139-244.

31 ONOZAWA, M. et al. Effects of soybean isoflavones on cell growth and apoptosis of the human prostatic cancer cell line LNCaP. Japanese Journal of Clinical Oncology, v. 28, n. 6, p. 360-363, 1998.

32 PARK, Y.K. et al. Biotransformação de isoflavonas de soja. Biotecnologia, Ciência e Desenvolvimento, v. 20, n. 5-6, p. $12-$ 14, 2001 .

33 PARK, Y.K.et al. Survey of isoflavone contents in Brazilian soybean. Ciencia y Tecnologia Alimentaria, v. 3, n. 2, p. 156-160, 2001b.

34 PARK, Y.K.; AGUIAR, C.L.; ALENCAR, S.M.; NERY, I.A.; SATO, H.H. Extraction of isoflavones from defatted soy flour by ethanol and conversion of glucoside isoflavones to aglycones by fungal betaglucosidase. In: IFT ANNUAL MEETING, 2001, New Orleans. Anais... New Orleans: IFT, 2001c. p. 164.

35 PETERSON, G.; BARNES, S. Genistein and biochanin A inhibit the growth of human prostate cancer cells but not epidermal growth factor receptor tyrosine autophosphorylation. Prostate, v. 22, p. 335-345, 1993.

36 POOL-ZOBEL, B.L. et al. Isoflavonoids and lignans have different potentials to modulate oxidative genetic damage in human colon cells. Carcinogenesis, v. 21, n. 6, p. 1247-1252, 2000.

37 SCHRAMM, D.D.; GERMAN, J.B. Potential effects of flavonoids on the etiology of vascular disease. Journal of Nutritional Biochemistry, v. 9, n. 10, p. 560-566, 1998.

38 SGARBIERI, V.C.; GARRUTI, E.C.; GUZMÁN, E.C. Soybeans as an extender of common beans. JAOCS, v. 58, n. 3, p. 522-526, 1981.

39 SHAHIDI, F.; WANASUNDARA, P. K. J. P. D. Phenolic antioxidants. Critical Reviews in Food Science and Nutrition, v. 32, n. 1, p. 67-103, 1992.

40 SHERTZER, H.G. et al. Inhibition of CYP1A1 enzyme activity in mouse hepatoma cell culture by soybean isoflavones. Chemico 
Biological Interactions, v. 123, n. 1, p. 31-49, 1999.

41 SKIBOLA, C.F.; SMITH, M.T. Potential health impacts of excessive flavonoid intake. Free Radical Biology and Medicine, v. 29, n. 34, p. 375-383, 2000.

42 VAN DER SCHOUW, Y.T. et al. Phyto-oestrogens and cardiovascular disease risk. Nutrition Metabolism and Cardiovascular Diseases, v. 10, n. 3, p. 154-167, 2000.

43 VERNETTI, F.J. História e importância da soja no Brasil. A Lavoura, v. 81, p. 21-24, 1977.

44 VIGNA, G.B. et al. Plasma lipoproteins in soy-treated postmenopausal women: a double-blind, placebo-controlled trial. Nutrition Metabolism and Cardiovascular Diseases, v. 10, n. 6, p. 315-322, 2000.

45 WANGEN, K.E. et al. Soy isoflavones improve plasma lipids in normocholesterolemic and mildly hypercholesterolemic postmenopausal women. American Journal of Clinical Nutrition, v. 73, n. 2, p. 225-231, 2001.

46 WOLF, W.D.; COWAN, J.C. Soybean as a protein source. London: Butterworth, 1971. $150 \mathrm{p}$.

47 WONG, C.K.; KEUNG, W.M. Bovine adrenal 3 beta-hydroxysteroid dehydrogenase/5-ene-4ene isomerase: characterization and its inhibition by isoflavones. Journal of Steroid Biochemistry and Molecular Biology, v. 71, n. 5-6, p. 191-202, 1999.

48 WONG, C.7K.; KEUNG, W.M. Daidzein sulfoconjugates are potent inhibitors of sterol sulfatase. Biochemical and Biophysical Research Communications, v. 233, n. 3, p. 579-583, 1997.

49 ZHENG, J. B.; RAMIREZ, V.D. Inhibition of mitochondrial proton F0F1ATPase/ATP synthase by polyphenolic phytochemicals. British Journal of Pharmacology, v. 130, n. 5, p. 1115-1123, 2000. 